\title{
Entropy
}

ISSN 1099-4300

www.mdpi.org/entropy/

Full paper

\section{CONVECTIVE HEATING OF SOLID SURFACE: ENTROPY GENERATION DUE TO TEMPERATURE FIELD AND THERMAL DISPLACEMENT}

\author{
Yagoub Nassar Al-Nassar \\ Mechanical Engineering Department, KFUPM, \\ P.O. Box 1876, Dhahran 31261, KSA. \\ ynassar@kfupm.edu.sa
}

Received: 11 October 2002 / Accepted: 6 July 2003 / Published: 31 December 2003

\begin{abstract}
Convective heating and cooling of the surfaces find application in process industry. During the heating or cooling cycle of the process, thermodynamic irreversibility which can be associated with the process parameters occurs. Moreover, thermodynamic irreversibility associated with the heating cycle can be quantified through entropy analysis. In the present study, convective heating of the solid surface is considered. A mathematical formulation of the temperature rise and thermal stress development during the transient heating process is presented. Entropy generation due to temperature field and thermal displacement is also formulated. The simulation for temperature rise, thermal displacement, and entropy generation are carried out for steel substrate. It is found that thermal displacement does not exactly follow the temperature distribution inside the substrate material. The magnitude of entropy generation due to temperature field is considerably higher than that corresponding to the thermal displacement.
\end{abstract}

Keywords: convective heating, thermal stress, thermal displacement, entropy generation 


\section{List of Symbols}

\begin{tabular}{|l|l|}
\hline$A$ & dimensionless parameter, $(\mathrm{c} / \alpha \gamma)$ \\
\hline$A_{C}$ & cross sectional area, $\mathrm{m}^{2}$ \\
\hline$c$ & thermal wave speed, $\mathrm{m} \mathrm{s}^{-1}$ \\
\hline$C_{v}$ & specific heat, $\mathrm{kJ} \mathrm{kg}^{-1} \mathrm{~K}^{-1}$ \\
\hline$E$ & Young's modulus, $\mathrm{Pa}$ \\
\hline$h$ & heat transfer coefficient, $\mathrm{W} \mathrm{m}^{-2} \mathrm{~K}^{-1}$ \\
\hline$k$ & thermal conductivity, $\mathrm{W} \mathrm{m}^{-1} \mathrm{~K}^{-1}$ \\
\hline$P$ & pressure, $\mathrm{Pa}$ \\
\hline$s$ & entropy, $\mathrm{kJ} \mathrm{kg}^{-1} \mathrm{~K}^{-1}$ \\
\hline$s^{*}$ & dimensionless entropy, $\left(\mathrm{s}^{*}=\mathrm{s} / \mathrm{C}_{\mathrm{v}}\right)$ \\
\hline$t$ & time, $\mathrm{s}$ \\
\hline$T$ & temperature, $\mathrm{K}$ \\
\hline$T_{0}$ & initial temperature, $\mathrm{K}$ \\
\hline$T^{*}$ & dimensionless temperature, $\left(T-T_{0}\right) /\left(\theta-T_{0}\right)$ \\
\hline$x$ & spatial coordinate along $\mathrm{x}$ axis, $\mathrm{m}$ \\
\hline$u$ & internal energy, $\mathrm{kJ} \mathrm{kg}^{-1}$ \\
\hline$\alpha$ & thermal diffusivity, $\mathrm{m}^{2} \mathrm{~s}^{-1}$ \\
\hline$\alpha_{T}$ & thermal expansion coefficient, $\mathrm{K}^{-1}$ \\
\hline$\beta$ & compressibility coefficient, $\mathrm{Pa}^{-1}$ \\
\hline$\gamma$ & $h / k, \mathrm{~m}^{-1}$ \\
\hline$\varepsilon$ & displacement, $\mathrm{m}$ \\
\hline$\varepsilon^{*}$ & dimensionless displacement \\
\hline$\varepsilon_{x}$ & strain \\
\hline$\theta$ & heat source temperature, $\mathrm{K}$ \\
\hline$v$ & Poisson's ratio \\
\hline$v$ & specific volume, $\mathrm{m}^{3} \mathrm{~kg}^{-1}$ \\
\hline$\rho$ & density, kg m \\
\hline$\sigma_{x}$ & stress, Pa \\
\hline
\end{tabular}

\section{Introduction}

Convective cooling of surface finds wide application in industry. In this case, cooling of the surface (i) improves mechanical and metallurgical properties of the substrate material, such as quenching, and (ii) enhances the life cycle of the equipment or parts such as cooling of electronic components. Moreover, during convective heating or cooling process, thermal stress is generated in the surface region of the 
substrate material. Depending on the temperature gradient in the region heated convectively, stress levels above or around yielding limit of the substrate material can occur. Consequently, investigation into thermal stress levels during the convective heating/cooling becomes necessary. Moreover, the temperature and stress fields are involved with thermodynamic irreversibility in the system. Therefore, study into thermodynamic irreversibility associated with the temperature and stress fields gives insight into the thermodynamic heating of the substrate material.

Considerable research studies were carried out to investigate the convective cooling/heating of the surfaces. Lumped capacitance analysis for the quenching of the metallic substrate was carried out by Campo and Hernandez-Morales [1]. They showed that for convective cooling, two primary dimensionless groups existed, which included solid fluid thermal conductivity ratio and modified Rayleigh number. The contact problems with frictional heat generation were studied by Kul'chyts'kyi Zhyhailo and Evtushenko [2] - for semi-bonded bodies of revolution by taking into account the effect of convective cooling of the free surface. They determined the conditions under which the influence of convective heat exchange on the level and distribution of contact stresses could be neglected. Numerical analysis of partially heated vertical plates in natural convective cooling was carried out by Campo et al [3]. They obtained the correlations between the induced mass flow rate as well as the maximum wall temperatures, the Rayleigh number, and the extension ratio. Transient two-dimensional thermal analysis of electronic packages by the boundary element method was examined by Guven et al [4 ]. They indicated that the results captured the singular flux field arising form the mismatched in the thermal conduction coefficients and geometric discontinuity. Three-dimensional convective cooling in a vertical channel with flush-mounted heat sources was carried out by Gunes and Laikopoulos [5]. They presented temperature and velocity field distributions as well as maximum temperature, maximum velocity, and local Nusselt number distributions in terms of Grashof number. Thermal characteristics of back cooled_impingement cooler for packaging of high power semiconductor laser diode array were studied by Mirji and Ahmad [6]. They showed that experimental thermal resistance values closely match with theoretical calculations.

Considerable research studies were carried out to explore thermal stresses developed in solid bodies. The numerical simulation of thermal stresses due to grinding was carried out by Moulik et al [7]. They indicated that the predicted thermal stress distributions were consistent with experimentally measured residual stresses on ground surface. Green's function for thermal stress mixed boundary value problem of an infinite plate was introduced by Hann and Hasebe [8]. They obtained an explicit solution including a hybergeometrical function by explaining the mapping technique and complex variable method. The thermal stress around a penny-shaped crack in a thermopiezoelectiric solid was studied by Shang and Kuna [9]. They presented exact expression for the stress-intensity factor. Thermal stress analysis and residual stresses in a thermoplastic composite beam were studied by Sayman et al [10]. They showed that for the same plastic distribution, the intensity of the transverse displacement was the 
largest for that orientation angle. Finite element modeling of stress development during deposition of ion assisted coating was carried out by Ward and Arnell [11]. They indicated that the predicted trends of mean stress and distribution were similar to those observed in the literature. Thermal stresses in an actively cooled two-piece rail structure were investigated by Satapathy and Persad [12]. They showed that use of thin resistive overlays minimized the thermal stresses on the structure.

Irreversibility of the thermal system can be quantified by the entropy analysis. A second law analysis of the optimum design and operation of thermal energy storage systems were considered by Robert et al [13]. He showed that an entire cycle of storage and removal should be considered when optimizing the design and performance of a thermal system. Thermodynamics analysis of thermo-mechanical coupling in Couette flow was studied by Demirel [14]. He indicated that since the irreversibility was related to loss of energy, the thermodynamic analysis could facilitate the optimal conditions which produced less entropy for an existing design. The second law analysis in fundamental convective heat transfer problems was carried out by Mahmud and Fraser [15]. They derived analytical expression for entropy generation and Bejan numbers from velocity and temperature profiles. Minimization of entropy generation by asymmetric convective cooling was investigated by Ibanez et al [16]. They showed that when the Biot numbers were different, the entropy function displayed a minimum for specific conditions. Yilbas [17] formulated entropy generation in nonequilibrium energy transport systems. He indicated that entropy generation enhanced as nonequilibrium energy transport increased across the two-nonequilibrium sub-systems.

Convective cooling of the surface and stress field due to temperature field were formulated previously [18]. However, the irreversibility associated with the system was left obscured. Consequently, the present study is conducted to present closed form solution for temperature and stress fields due to convective heating of the surface and previously obtained solution for the temperature and stress distributions are enhanced. Entropy generation due to temperature and stress fields is formulated and the magnitude of entropy generation is predicted for various heat transfer coefficients.

\section{Mathematical Model}

Consider a semi-infinite solid body heated by convection heat transfer at the surface as shown in Figure 1. A steep temperature gradient is generated in the surface region of substrate material during this process. The problem of thermal shock on solid surface can be examined in half space. The closed form solution of the unsteady temperature field $T(x, t)$ in the half space takes the form, as given by Shuja and Yilbas [18 ], 


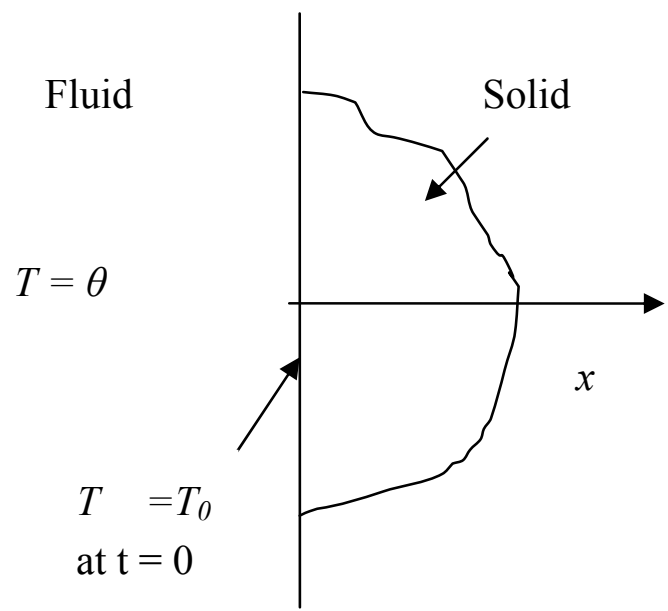

Figure 1 A schematic view of heating situation.

$\frac{T-T_{0}}{\theta-T_{0}}=\operatorname{erfc}\left(\frac{x}{2 \sqrt{\alpha t}}\right)-\exp \left(\gamma x+\gamma^{2} \alpha t\right) \cdot \operatorname{erfc}\left(\frac{x}{2 \sqrt{\alpha t}}+\gamma \sqrt{\alpha t}\right)$

where $T_{o}, \theta, \alpha$, and $\gamma$ are the initial surface temperature $(x=0, t=0)$, heat source temperature, thermal diffusivity, and the ratio of the heat transfer coefficient $(h)$ to the thermal conductivity $(k)$, respectively. erfc stands for the complementary error function, which is 1- erf.

From the stress analysis of one dimensional thermoelasticity problem, the thermal strain, $\varepsilon_{\mathrm{x}}$, in a solid substrate along the $\mathrm{x}$ axis as a function of the stress, $\sigma_{x}$, and temperature, $\Delta T=\left(T-T_{o}\right)$, can be written as

$\varepsilon_{x}=\frac{(1+v)(1-2 v)}{(1-v) E} \sigma_{x}+\frac{(1+v)}{(1-v)} \alpha_{\mathrm{T}} \Delta T$

where $v, E$, and $\alpha_{\mathrm{T}}$ are the Poisson's ratio, elastic modulus, and thermal expansion of a solid material, respectively.

Upon solving the equation of motion referenced to [18] the closed form solution of the stress field for which the surface $(x=0)$ is stress free as well as when $x$ is very large $(x=\infty)$ is given as 


$$
\sigma_{x}= \begin{cases}\sigma_{x 1} & 0<t<\frac{x}{c} \\ \sigma_{x 2} & t>\frac{x}{c}\end{cases}
$$

where

$$
\begin{gathered}
\sigma_{x 1}=\frac{E \alpha_{\mathrm{T}}\left(\theta-T_{o}\right)}{1-2 v}\left\{\frac{1}{2\left(1+\frac{c}{\alpha \gamma}\right)} \exp \left[\frac{c^{2}}{\alpha}\left(t-\frac{x}{c}\right)\right] \cdot \operatorname{erfc}\left(\frac{x}{2 \sqrt{\alpha t}}-c \sqrt{t / \alpha}\right)-\frac{1}{2\left(1-\frac{c}{\alpha \gamma}\right)} \cdot\right. \\
\left.\exp \left[\frac{c^{2}}{\alpha}\left(t+\frac{x}{c}\right)\right] \cdot \operatorname{erfc}\left(\frac{x}{2 \sqrt{\alpha t}}-c \sqrt{t / \alpha}\right)-\frac{1}{1-\frac{c^{2}}{\alpha^{2} \gamma^{2}}} \exp \left(\gamma x+\gamma^{2} \alpha t\right) \cdot \operatorname{erfc}\left(\frac{x}{2 \sqrt{\alpha t}}+\gamma \sqrt{\alpha t}\right)\right\}
\end{gathered}
$$

(4)

$$
\begin{gathered}
\sigma_{x 2}=\frac{E \alpha_{\mathrm{T}}\left(\theta-T_{0}\right)}{1-2 v} \cdot \frac{1}{1-\frac{c^{2}}{\alpha^{2} \gamma^{2}}}\left\{\exp \left[\frac{c^{2}}{\alpha}\left(t-\frac{x}{c}\right)\right] \cdot\left\{1-\frac{c}{\alpha \gamma} \operatorname{erf}\left[\frac{\mathrm{c}^{2}}{\alpha}\left(t-\frac{x}{c}\right)\right]^{\frac{1}{2}}\right\}\right. \\
\left.-\exp \left[\alpha \gamma^{2}\left(t-\frac{x}{c}\right)\right] \cdot \operatorname{erfc}\left(\gamma\left[\alpha\left(t-\frac{x}{c}\right)\right]^{\frac{1}{2}}\right)\right\}
\end{gathered}
$$

where $c=E(1-v) /[(1+v)(1-2 v) \rho]$ is the velocity of propagation of elastic longitudinal wave and $\rho$ is the density of the solid material.

In the case of entropy calculations, entropy generation in the solid is a function of temperature and volume change due to thermal expansion, in this case $s=s(T, v)$, therefore one can write

$d s=M d T+N d v$

where $\quad M=\left(\frac{\partial S}{\partial T}\right)_{v}$ and $N=\left(\frac{\partial S}{\partial v}\right)_{T} \cdot d v$

By definition:

$\alpha_{T}=-\frac{1}{v}\left(\frac{\partial v}{\partial T}\right)_{P}$ and $\beta=-\frac{1}{v}\left(\frac{\partial v}{\partial P}\right)_{T}$ 
where $\alpha_{T}$, and $\beta$ are thermal expansion coefficient and compressibility coefficient, respectively.

Considering the Helmholtz function, $F=u-T \mathrm{~s}$

and

$$
d F=d u-T d s-s d T,
$$

and knowing that $\quad d u=T d s-P d v$,

therefore $\quad d F=-s d T-P d \mathrm{~s}$.

Using the following Maxwell relation

$-\left(\frac{\partial s}{\partial v}\right)_{T}=-\left(\frac{\partial P}{\partial T}\right)_{v}$

one can reach to the relation

$\left(\frac{\partial s}{\partial v}\right)_{T}=-\frac{\alpha_{T}}{\beta}$

Also, the relation: $d u=T d s-P d v$ reduces to

$\left(\frac{\partial u}{\partial T}\right)_{v}=T \cdot\left(\frac{\partial s}{\partial T}\right)_{v}$ and knowing that $\left(\frac{\partial u}{\partial T}\right)_{v}=C_{v}$

Therefore $\left(\frac{\partial s}{\partial T}\right)_{v}=\frac{C_{v}}{T}$

Now equation (6) becomes

$d s=\frac{C_{v}}{T} \cdot d T-\frac{\alpha_{T}}{\beta} \cdot d v$

which is a unique solution for entropy generation in solid obtained from Maxwell relations.

Looking at the second term in equation (10)

$v \cdot \frac{\alpha_{T}}{\beta} \cdot \frac{d v}{v}=v \cdot \frac{\alpha_{T}}{\beta} \cdot \frac{A_{c} d \varepsilon}{A_{c} \varepsilon}=\frac{1}{\rho} \cdot \frac{\alpha_{T}}{\beta} \cdot \frac{d \varepsilon}{\varepsilon}$ 
where ( $v=\frac{1}{\rho}$, specific volume and $\varepsilon$ displacement ). Therefore

$d s=C_{v} \cdot \frac{d T}{T}-\frac{1}{\rho} \cdot \frac{\alpha_{T}}{\beta} \cdot \frac{d \varepsilon}{\varepsilon}$

Equation (11) can be written in non-dimensional form by introducing the following non-dimensional parameters

$$
\begin{aligned}
& s^{*}=s / C_{v} \\
& A=\frac{1}{\rho C_{v}} \cdot \frac{\alpha_{T}}{\beta} \\
& d s^{*}=\frac{d T^{*}}{T^{*}}-A \cdot \frac{d \varepsilon^{*}}{\varepsilon^{*}}
\end{aligned}
$$

Integrating equation (12) yields:

$$
\Delta s^{*}=\ln \left(\frac{T_{1}^{*}}{T_{o}^{*}}\right)-A \cdot \ln \left(\frac{\varepsilon_{1}^{*}}{\varepsilon_{o}^{*}}\right)
$$

The displacement, $\varepsilon$, can be obtained after integration of equation (2), i. e.:

$\varepsilon=\int_{0}^{\infty} \varepsilon_{x} d x$

A mathematica software is used to compute temperature, displacement, and entropy generation during convective heating of the surface.

\section{Results and Discussions}

Heating of solid substrate due to convective boundary at the surface is formulated. The closed form solution for the thermal stress field is presented. The entropy analysis due to temperature and stress fields are carried out to demonstrate the temperature, displacement, and entropy fields, steel is employed in the simulation.

Figure 2 shows temperature profiles inside the substrate material for three different heat transfer coefficients. The sharp increase of the temperature occurs in the surface region of the substrate material, which is particularly true for high heat transfer coefficients $\left(\mathrm{h}>10^{5} \mathrm{~W} / \mathrm{m}^{2} \mathrm{~K}\right)$. Temperature decay along the depth appears to be almost linear despite the fact that the heating situation is transient. This is because of: (i) long period of heating duration, steady heating situation is resulted, and (ii) low 
heat transfer coefficient ( $\mathrm{h} \leq 10^{7} \mathrm{~W} / \mathrm{m}^{2} \mathrm{~K}$ ); i. e., impulsive like heating (shock) at the surface is omitted. The influence of heat transfer coefficient on the temperature rise is considerable, provided that high heat transfers coefficient $\left(10^{7} \mathrm{~W} / \mathrm{m}^{2} \mathrm{~K}\right)$ results in rapid change of temperature gradient in the substrate material, i.e. temperature gradient reduces with progressing heating duration.

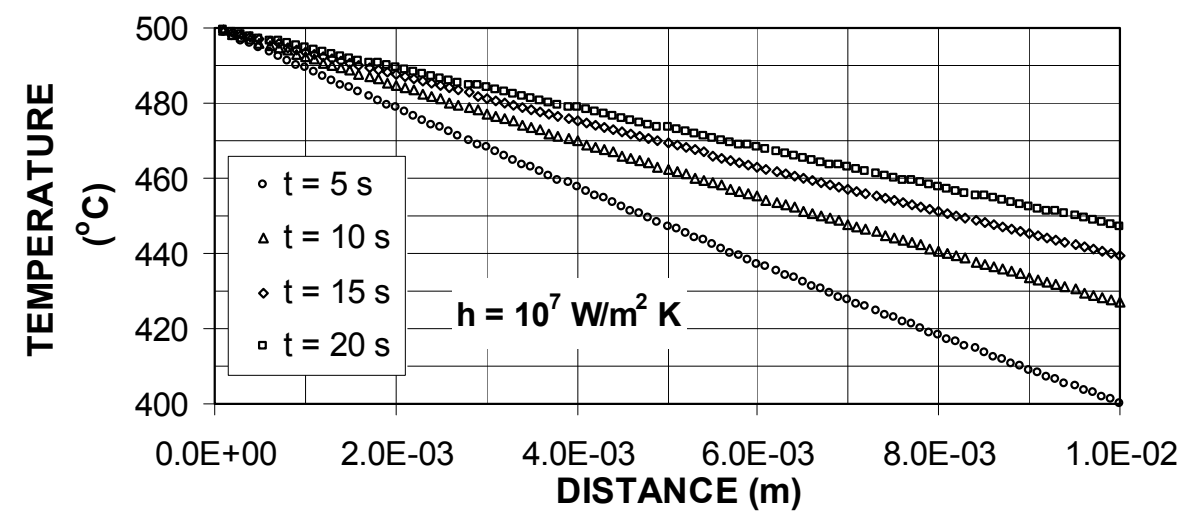

(a)

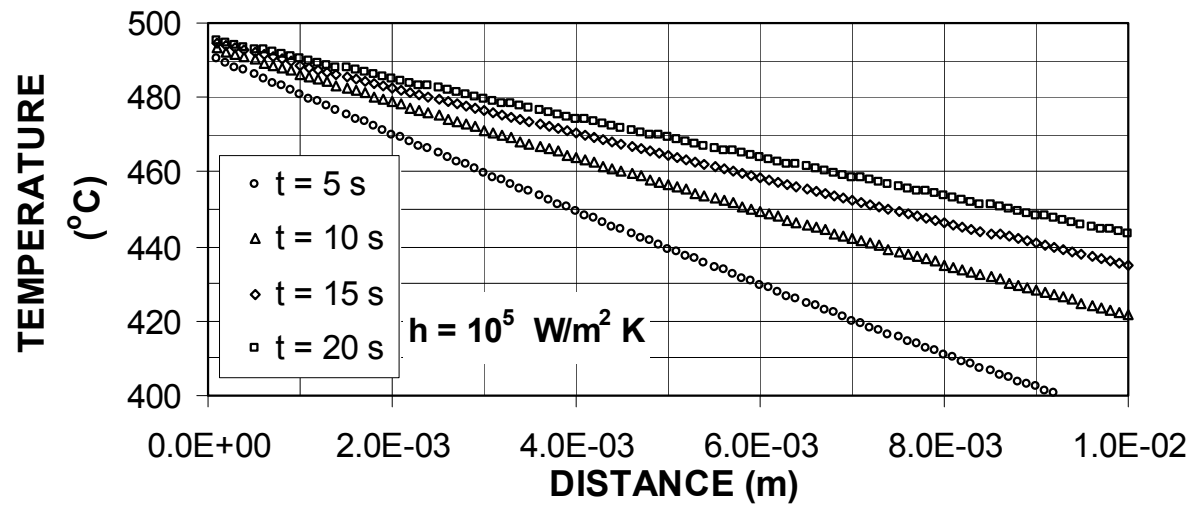

(b)

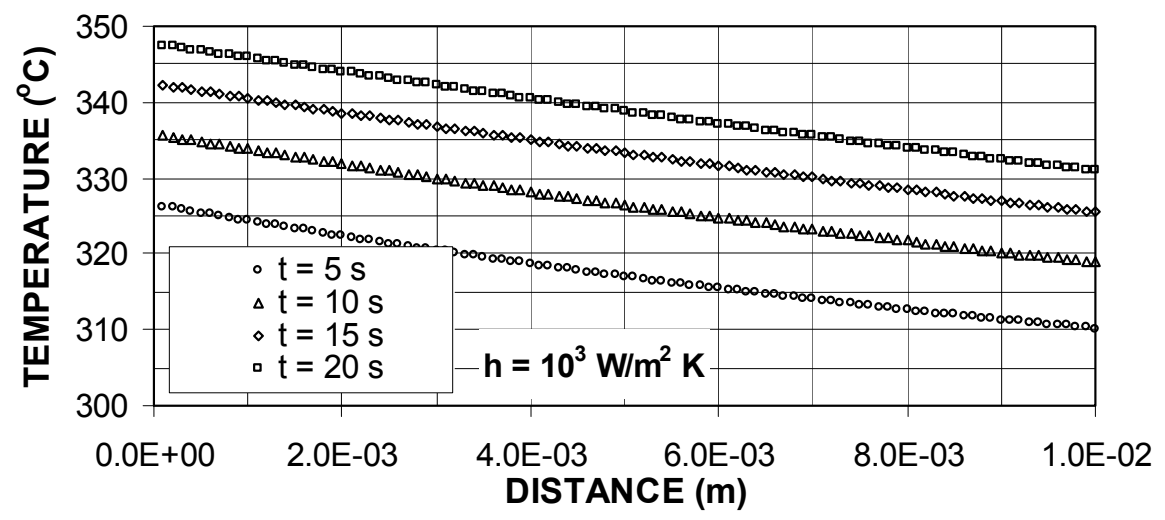

(c)

Figure 2 Temperature inside the substrate material for different heating periods for three different 
values of convective heat transfer coefficients, namely 103,105, and $107 \mathrm{~W} / \mathrm{m} 2 \mathrm{~K}$.

Figure 3 shows the thermal displacement inside the substrate material for different heating durations. The displacement is maximum at the surface and almost zero at some depth below the surface. The thermal displacement does not follow exactly the temperature profiles despite the fact that the substrate material is considered to be elastic. This is because of the strain developed in the surface region which does not vary linearly along the depth like temperature profiles, i.e. expansion of the substrate material below the surface is suppressed by the thermal strain developed in this region. As similar to temperature behavior, increasing heat transfer coefficient results in large magnitude of displacement particularly at the surface of substrate material. The displacement gradient attains slightly higher values at low heat transfer coefficients as compared to that corresponding to high heat transfer coefficients. This is due to the temperature profiles, provided that the variation in temperature gradient is larger than its counterpart corresponding to the displacement.

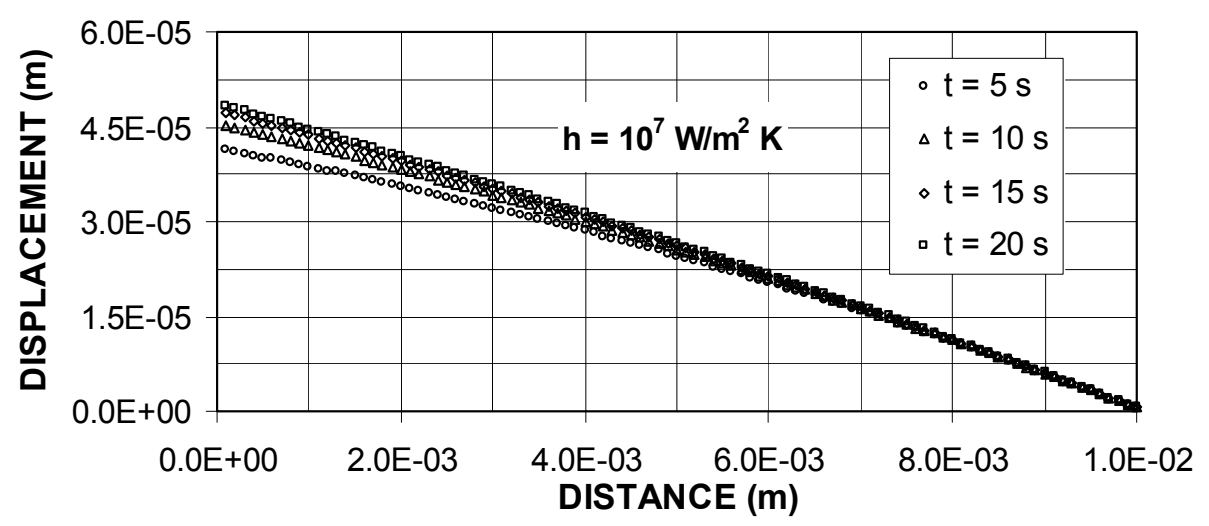

(a)

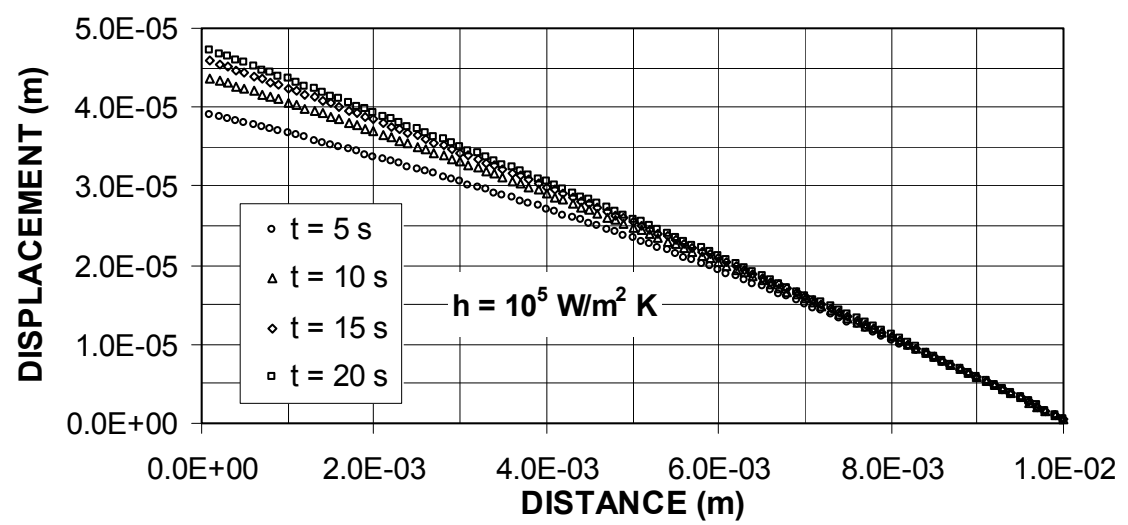

(b) 


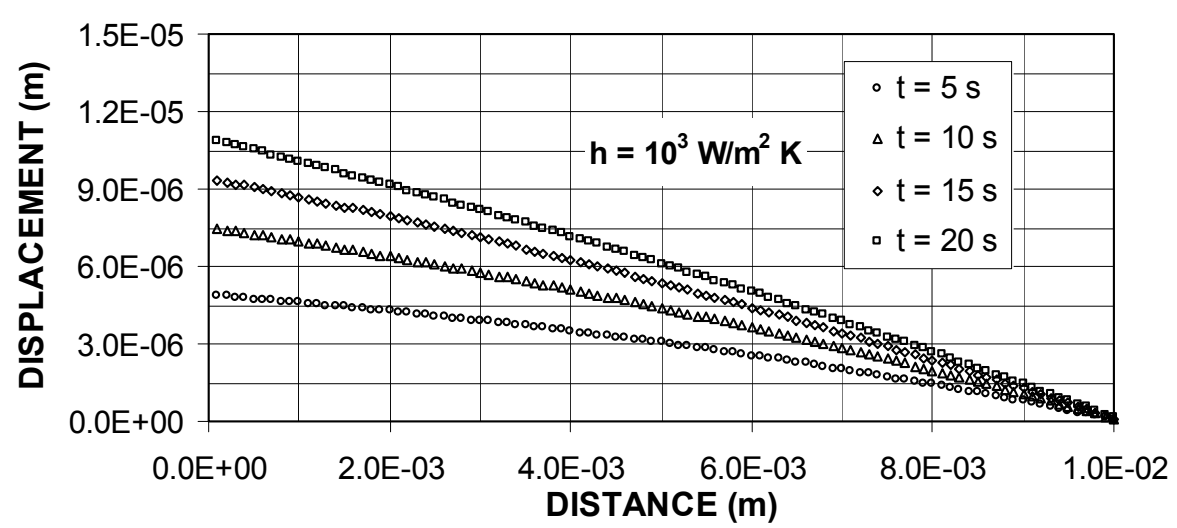

(c)

Figure 3: Displacement inside the substrate material for different heating periods for three different values of convective heat transfer coefficients, namely 103,105 , and $107 \mathrm{~W} / \mathrm{m} 2 \mathrm{~K}$.

Entropy analysis covers the entropy generation due to temperature field and thermal strain. However, thermal strain can be formulated through thermal displacement. Consequently, equation (13) is written in terms of temperature field and thermal displacement. Therefore, entropy generation due to temperature field and thermal displacement are segregated. Figure 4 shows entropy generation due to temperature field inside the substrate material for different heating periods. Entropy attains maximum value in the surface vicinity and as the distance increases away from the surface it reduces. The entropy profiles follow almost the temperature profiles provided that the slopes of both curves differ. In the early heating periods entropy production is low and as the heating duration progresses, it increases. Moreover, for high heat transfer coefficients $\left(\mathrm{h} \geq 10^{5} \mathrm{~W} / \mathrm{m}^{2} \mathrm{~K}\right)$, entropy production increases. This is because of the heat transfer rate to the solid material, which increases with improving heat transfer coefficients.

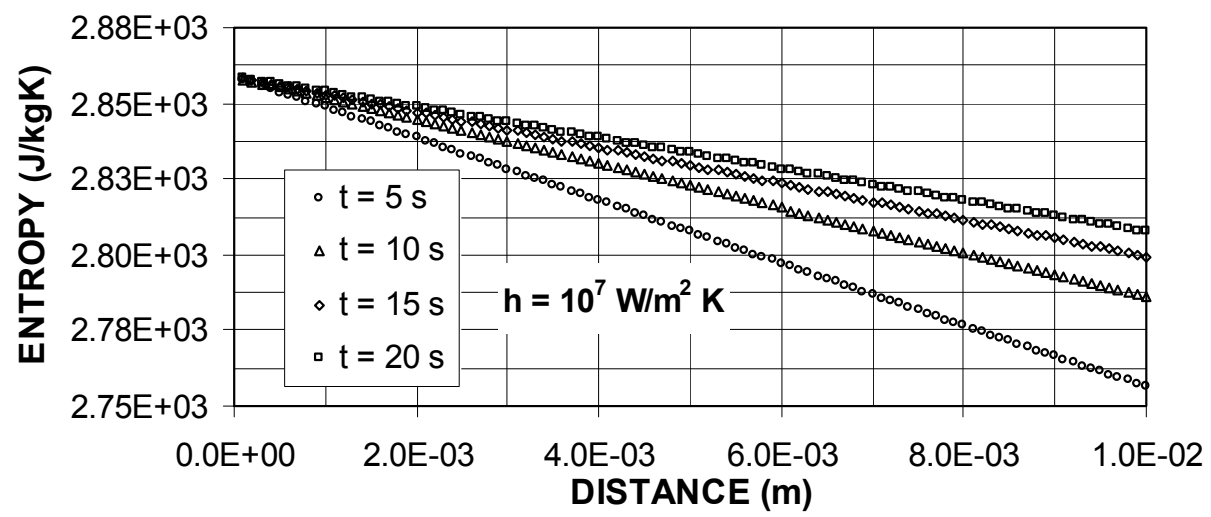

(a) 


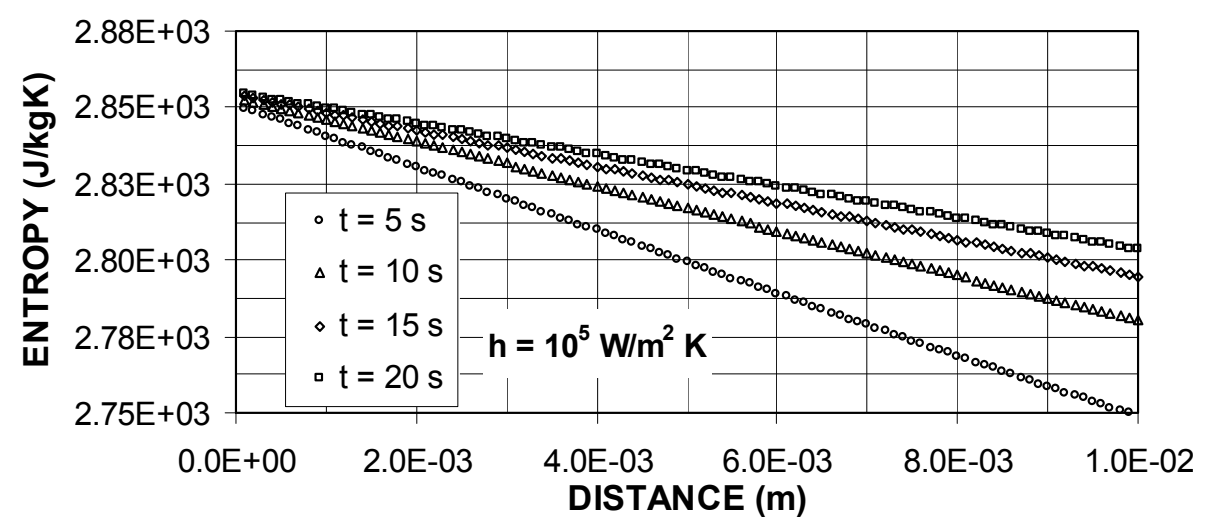

(b)

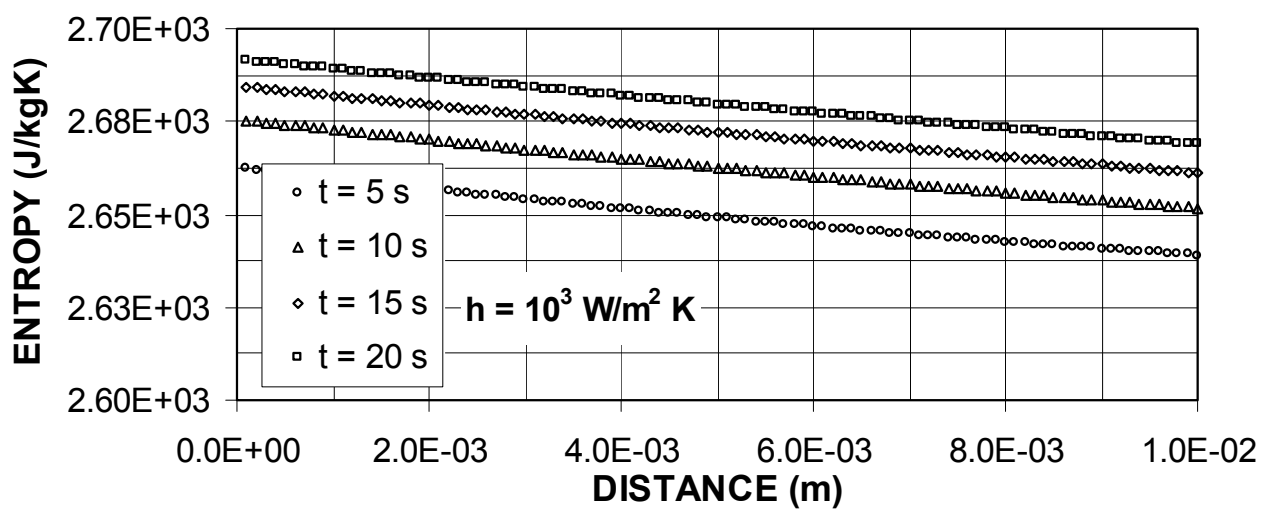

(c)

Figure 4:Entropy generation due to temperature field inside the substrate for three different values of convective heat transfer coefficients, namely 103,105, and $107 \mathrm{~W} / \mathrm{m} 2 \mathrm{~K}$.

Figure 5 shows entropy generation due to the stress field inside the solid substrate for different heating periods. Entropy generation attains high values in the surface region and it reduces with increasing depth below the surface. Entropy profiles follow almost the displacement profiles, provided that their magnitudes are different. Moreover, entropy generation increases with progressive heating periods. This occurs because of the thermal strain, which enhances with progressive heating period. Entropy generation also increases with increasing heat transfer coefficient. In this case increasing heat transfer rates at the surface enhances the displacement in the surface region; consequently, entropy generation increases in this region. When comparing figures 4 and 5, it can be observed that entropy generation due to temperature field attains considerably higher values in the surface region as compared to its counterpart corresponding to stress field. This indicates that entropy generated due to internal energy gain of the substrate material dominates over the mechanical work done during the displacement of the surface. 


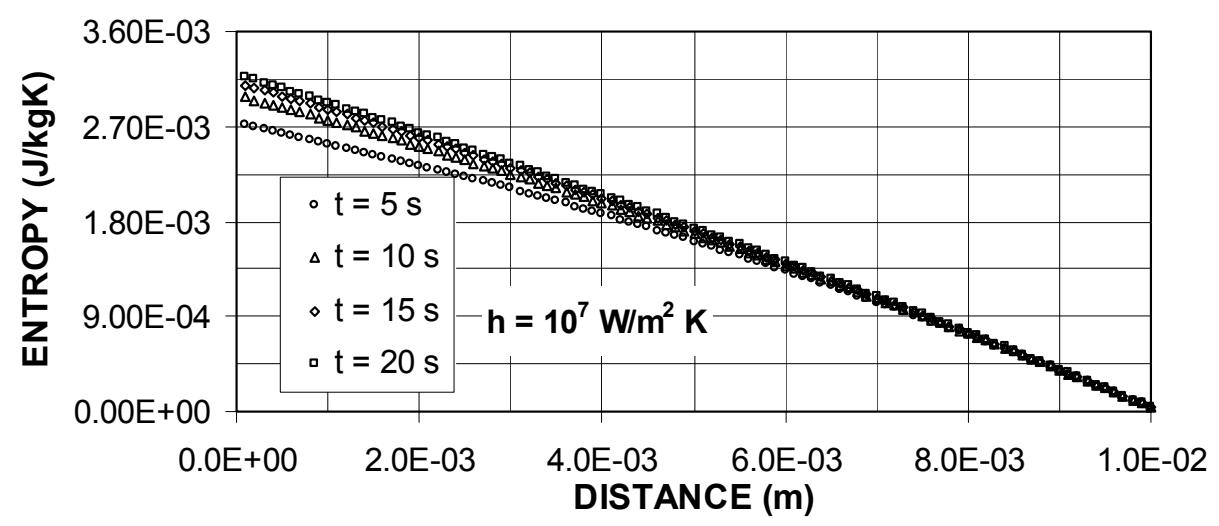

(a)

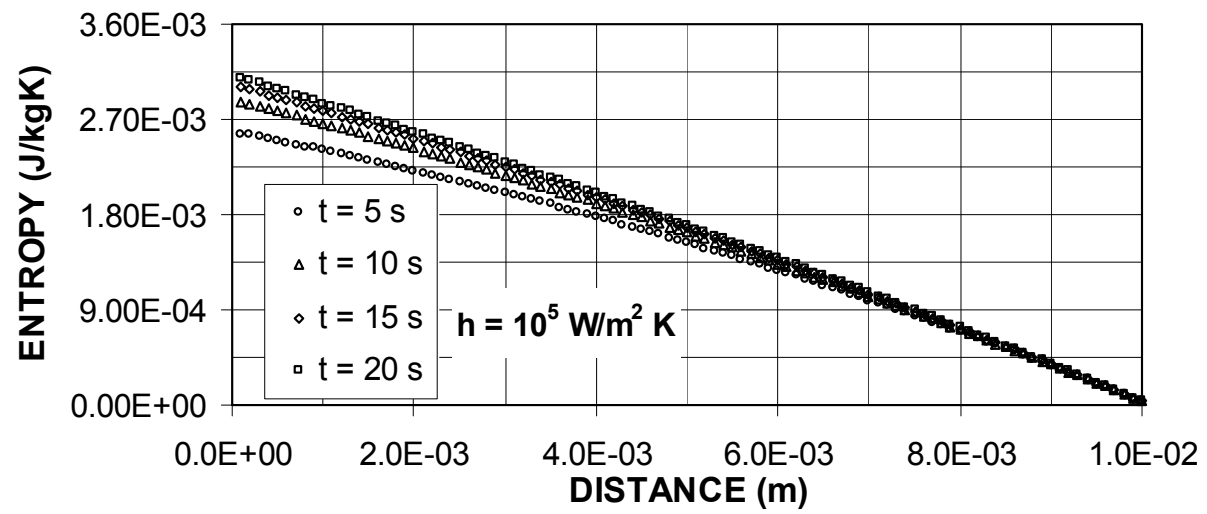

(b)

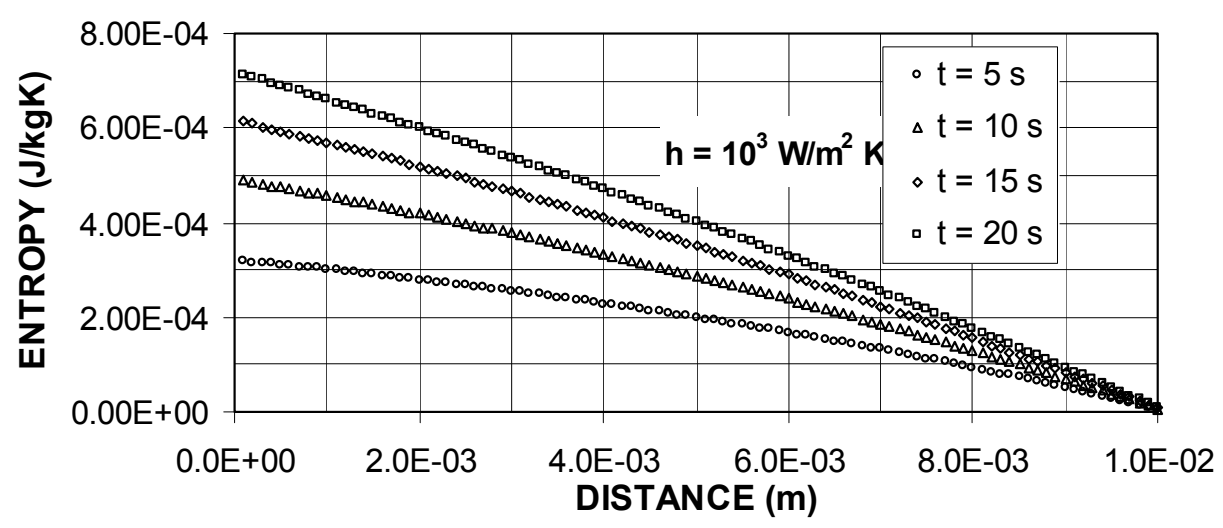

(c)

Figure 5:Entropy generation due to stress field inside the substrate for three different values of convective heat transfer coefficients, namely 103,105, and $107 \mathrm{~W} / \mathrm{m} 2 \mathrm{~K}$. 


\section{Conclusion}

Temperature and stress fields due to convective heating of the solid substrate at surface are formulated analytically. The irreversibility associated with the thermal system is quantified through the entropy generation. Steel is employed to simulate the temperature field and thermal displacement during the convective heating process. It is found that temperature gradient is high in the surface region of the substrate material in the early heating period, which is more pronounced at high transfer coefficients. Thermal displacement does not follow the temperature profiles, which is due to thermal strain developed in the region close to the surface. In this case, thermal strain varies with temperature gradient. Thermal displacement increases when both heat transfer coefficient and heating period increase. Entropy generated due to temperature field follows almost temperature profiles, provided that the magnitudes of the slopes of both curves are different. Entropy generation in surface vicinity is higher than that corresponding to some depth below the surface. Entropy generation due to stress field follows thermal displacement profiles, provided that the magnitudes of both curves differ. When comparing entropy generation due to temperature and stress fields, it can be observed that entropy generation due to temperature field is considerably higher than that corresponding to stress field. This indicates that internal energy gain of the substrate material dominates over the mechanical work done due to thermal displacement of the surface during the heating process.

\section{Acknowledgments}

The authors acknowledge the support of the King Fahd University of Petroleum and Minerals, Dhahran, Saudi Arabia for this work.

\section{References}

1. Campo A. and Hernandez-Morales B., Lumped-capacitance analysis for the quenching of workpieces: Limitations in terms of Rayleigh numbers and solid/fluid thermal conductivity ratios, Journal of Materials Processing \& Manufacturing Science, 1997, 5(4): 295-301.

2. Kul'chyts'kyi-Zhyhailo R. D. and Evtushenko O. O., Convective cooling in contact problems of thermoelasticity with frictional heat generation, Materials Science, 1997, 33 (6): 818-824.

3. Campo A., Manca O., and Morrone B., Numerical analysis of partially heated vertical parallel plates in natural convective cooling, Numerical Heat Transfer Part A-Applications, 1999, 36(2): 129-151.

4. Guven I., Chan C.L., and Madenci E., Transient two-dimensional analysis of electronic packages by the boundary element method, IEEE Transactions on Advanced Packaging, 1999 22(3): 476-486. 
5. Gunes H. and Liakopoulos A., Three-dimensional convective cooling in a vertical channel with flush-mounted heat sources, International Journal of Heat and Mass Transfer, 2003, 46(5): 791-808.

6. Mirji S. A. and Ahmad S., Thermal characteristics of back cooled impingement cooler for packaging of high power semiconductor laser diode arrays, IETE Journal of Research, 2002, 48(6): 437-440.

7. Moulik P. N., Yang H.T.Y., and Chandrasekar S., Simulation of thermal stresses due to grinding, International Journal of Mechanical Sciences, 2001, 43(3): 831-851

8. Han J. J. and Hasebe N., Green's function for thermal stress mixed boundary value problem of an infinite plane with an arbitrary hole under a point heat source, Journal of Thermal Stresses, 2002, 25(12): 1147-1160.

9. Shang F. and Kuna M., Thermal stress around a penny-shaped crack in thermopiezoelectric solid, Computational Materials Science, 2003, 26: 197-201.

10. Sayman O., Belevi M., and Duranaya M., Thermal stress analysis and residual stresses in thermoplastic composite beam, Journal of Reinforced Plastic and Composites, 2003, 22(1): 67-81.

11. Ward D. J. and Arnell R. D., Finite element modeling of stress development during deposition of ion assisted coatings, Thin Solid Film, 2002, 420: 269-274.

12. Satapathy S. and Persad C., Thermal stresses in actively cooled two-piece rail structure, IEEE Transactions on Magnetics, 2003, 39(1): 468-471.

13. Robert J. K., A second law analysis of the optimum design and operation of thermal energy storage systems, International Journal of Heat Transfer, 1987, 30(1): 43-57.

14. Demirel Y., Thermodynamic analysis of thermomechanical coupling in Couette flow, International Journal of Heat Transfer, 2000, 43: 4205-4212.

15. Mahmud S. and Fraser R. R., The second law analysis in fundamental convective heat transfer problems, International Journal of Thermal Sciences, 2003, 42(2): 177-186.

16. Ibanez G., Cuevas S., and de Haro M. L., Minimization of entropy generation by asymmetric convective cooling, International Journal of Heat and Mass Transfer, 2003, 46(8): 1321-1328.

17. Yilbas B. S., Entropy production during laser picoseconds heating of copper, ASME, Journal of Energy Resources Technology, 2002, 124: 204-213.

18. Shuja S. Z. and Yilbas B. S., Thermal stresses owing to convective heating at surface, Surface Engineering, 2002, 18(3): 202-207.

(C) 2003 by MDPI. (http://www.mdpi.org). Reproduction for noncommercial purposes permitted. 